\title{
Metodología para el desarrollo de proyectos de intervención en la línea terminal de comunicación organizacional de la UPAEP
}

\section{Methodology for the development of intervention projects in the UPAEP organizational communication terminal line}

Eva María Pérez Castrejón*

Universidad Popular Autónoma del Estado de Puebla (UPAEP)

21 Sur núm.1103, Col. Santiago, C.P. 72410, Puebla, Puebla

Carmen Karina Torrescano de la Peña**

Universidad Popular Autónoma del Estado de Puebla (UPAEP)

21 Sur núm.1103, Col. Santiago, C.P. 72410, Puebla, Puebla

\section{Editor: Rogelio del Prado Flores}

Fecha de recepción: 15 de abril de 2020

Fecha de aceptación: 9 de mayo de 2020 evamaria.perez@upaep.mx

https://orcid.org/0000-0002-3295-3901

carmenkarina.torrescano@upaep.mx https://orcid.org/0000-0003-4739-6199

https://doi.org/10.36105/stx.2020n5.04

\section{RESUMEN}

El presente artículo muestra la metodología que se ha utilizado para el desarrollo de proyectos de intervención en la línea terminal de comunicación organizacional de la Universidad Popular Autónoma del Estado de Puebla (UPAEP), además se presentan diferentes aportes teóricos que sirven de apoyo para argumentar el modelo aplicado en los diferentes proyec-

\footnotetext{
* Maestra en Procesos de Diseño por la Universidad Popular Autónoma del Estado de Puebla (UPAEP) y Doctora en Comunicación Aplicada por la Universidad Anáhuac México, Ciudad de México. Experiencia académica por más de dieciocho años. Dirige actualmente los Proyectos Estratégicos y Trasversales de la Vicerrectoría de Investigación y Posgrados. Estancia de investigación en la Universidad de Málaga, España. Asesora de proyectos de comunicación interna para organizaciones públicas y privadas; ha impartido talleres, pláticas y cursos para diferentes universidades del estado y el país. Ha sido parte de los comités coordinadores o ha realizado algún tipo de actividad en CONEICC, AMCO, AMIPCO, CIEES, CENEVAL, CONAC, entre otros.

** Licenciada en Ciencias de la Comunicación y Maestra en Educación por la Universidad Popular Autónoma del Estado de Puebla (upaep). Actualmente es profesora de tiempo completo del programa de la licenciatura en Comunicación y Medios Digitales de la misma institución. Se ha desempeñado como Coordinadora de la Línea Terminal de Comunicación Organizacional. Es miembro de la Asociación Mexicana de Comunicadores (AMCO) y ha participado como par evaluador en Procesos de Acreditación de Programas de Comunicación por parte del Consejo de Acreditación de la Comunicación (CONAC). Ha trabajado con organizaciones asesorando proyectos de comunicación interna y desarrollo organizacional.
} 
tos de intervención que realizan los estudiantes de esta casa de estudios en organizaciones públicas y privadas como una forma de vincular su formación en la línea con sus prácticas profesionales y experiencia laboral, además de reforzar el desarrollo teórico en congruencia con la práctica universitaria.

Palabras clave: Comunicación organizacional, proyecto de intervención, línea terminal, UPAEP.

\begin{abstract}
In this article shows the methodology that has been used for the development of intervention projects in the terminal line of organizational communication of the Universidad Popular Autónoma del Estado de Puebla (UPAEP), in addition there are different theoretical contributions that serve as support to argue the model applied in the different intervention projects carried out by the students of this house of studies in public and private organizations as as a way to link their training in line with their professional practices and work experience, in addition to reinforcing theoretical development consistent with university practice.
\end{abstract}

Keywords: Organizational communication, intervention project, terminal line, UPAEP.

\title{
INTRODUCCIÓN
}

L a comunicación organizacional se ha ido fortaleciendo a través del tiempo como una disciplina cuyas directrices han variado, las cuales se han sustentado y argumentado desde la investigación y las diferentes propuestas teóricas. Dentro de la comunicación organizacional se cuenta con una riqueza de aportaciones que han permitido la construcción del campo de la comunicación y por tanto el diseño, aplicación y evaluación de proyectos de intervención que buscan contribuir positivamente en las organizaciones públicas y privadas mejorando los procesos de comunicación.

La metodología que se aplica en los proyectos de intervención que diseñan estudiantes de la línea terminal en Comunicación Organizacional (CO) de la UPAEP es a partir de diferentes bases teóricas que han dado caminos adicionales hacia la investigación aplicada con el propósito de que sus esfuerzos de reflexión ayuden a la solución de problemáticas y/o áreas de oportunidad que hay en los diferentes sistemas organizacionales. De acuerdo con lo que establecen Tanius Karam y Andrés Cañizáles (20I0), la comunicación organizacional necesariamente debe percibirse de manera integral y no reduccionista como medios de 
comunicación internos. Es necesario que el especialista de la Co haga una reflexión y crítica permanentes de las circunstancias internas y externas e investigue cuáles son las necesidades reales y así diseñar y proponer las mejores estrategias de comunicación.

La comunicación organizacional posibilita que las organizaciones tanto públicas como privadas cumplan con los objetivos que se establecen, la línea terminal de la UPAEP propone que esto sea posible a través de un diagnóstico, posteriormente una propuesta estratégica y para concluir una evaluación de resultados. Es necesaria la innovación en las organizaciones como un tema de gran interés en el espacio académico, donde su percepción conceptual se aborda de maneras diferentes (Rodríguez, R., Alburquerque y Contreras, 2013). Los estudiantes del programa académico en Comunicación, de la línea terminal en CO de la UPAEP desarrollan competencias y obtienen los conocimientos necesarios que posibilitan analizar las organizaciones públicas o privadas, y así decidir las acciones de comunicación organizacional que puedan incidir favorablemente en los públicos internos y externos.

El trabajo presenta cronológicamente cómo se aplican en organizaciones públicas y privadas desde la metodología los proyectos de intervención con una perspectiva cuantitativa, lo que ha permitido el diseño, aplicación y evaluación de proyectos de intervención que llevan implícitos planes estratégicos soportados por diferentes piezas de comunicación. Los estudiantes de la UPAEP deciden qué línea estudiar desde el fenómeno de la comunicación, a partir del séptimo semestre, la incidencia y obtención de conocimientos de la disciplina es mayor; obtienen diferentes conocimientos y perspectivas, examinan los contextos que prevalecen en los entornos organizacionales.

\section{SUSTENTO TEÓRICO}

Esta investigación favorece a las instituciones de educación superior que ofertan la licenciatura en Comunicación con línea disciplinar en comunicación organizacional y que están interesadas en que sus estudiantes combinen la teoría y la práctica a través de proyectos de intervención que pueden aplicar en organizaciones públicas y privadas. Esto, gracias al diseño de planes estratégicos, los cuales previamente tuvieron un proceso de diagnóstico que permite identificar las herramientas de comunicación necesarias. Asimismo, esta investigación impulsa a que a través de los programas académicos se propongan y elaboren contenidos teórico-prácticos que respondan a las necesidades organizacionales como una contestación social. 


\section{LA COMUNICACIÓN ORGANIZACIONAL, DISCIPLINA \\ INELUDIBLE PARA LAS ORGANIZACIONES}

La comunicación en las organizaciones se ha propuesto desde su origen a dar una respuesta a la comunidad, por lo que se parte de una definición clásica como lo es la de Pasquali (1978), quien afirma que la comunicación aparece cuando la sociedad se estructura formalmente y sin esta no habría estructura. La comunicación se manifiesta como el máximo signo de expresión de los individuos, a través de la cual puede vincular relaciones humanas efectivas mediante signos que estructuran mensajes y que establecen significados en común por medio de un proceso participativo de un emisor y un receptor. El objetivo principal de la comunicación es persuadir, convencer al otro con el uso de la razón.

Por lo que la comunicación se convierte en un proceso importante para las organizaciones, de acuerdo con referentes teóricos se estudia como tal desde I950, aunque para Charles Redding es desde 1972. Para las organizaciones, la comunicación se ha convertido en el canal que posibilita las relaciones interpersonales efectivas en las interacciones labores diversas.

Es importante hacer una pausa y definir a la organización como la "unidad social con objetivos específicos" (Ramió y Ballart, I993, p. 2I) o como el contexto donde los seres humanos se pueden relacionar de manera solidaria y conformar sistemas que pueden responder ante las perturbaciones de los contextos. "La organización es un todo, en su dinámica, genera nuevos efectos a las partes que la constituyen” (Morín, 2005, p. I8I).

Retomando el desarrollo histórico de la disciplina se menciona que en la década de los años cincuenta la comunicación organizacional era estudiada por los especialistas y la veían como un sistema general y abierto (Von Bertalanffy, 1995, p. Io). Para 1960 Elton Mayo aporta nuevos estudios, en donde las organizaciones se ven como sistemas cerrados. El especialista implementa estrategias para defender la imagen de los líderes (Bland, I992). En la década de los años setenta, para la comunicación organizacional hubo un número considerable de investigaciones; el estudio se centraba en el clima organizacional. Federico Varona en 1993 comentó que fue crucial el desarrollo de las auditorías y que de acuerdo con Gerald Goldhaber (1987) estas incluían métodos de evaluación (cuestionarios, entrevistas, análisis de redes de información, experiencias críticas y un diario de comunicación) (Varona, 1994). En estos años, en América Latina la comunicación organizacional adquiere mayor relevancia ante los procesos de industrialización (León, 20II). En la década de los años ochenta aparecen las primeras publicaciones de las auditorías de comunicación organizacional (Varona, 1994, p. 13), también de acuerdo con Thomas B. Jablin (1990) los investigadores se enfocan al estudio de los procesos de la comunicación 
y de los procesos organizacionales. Conjuntamente fueron años de centrar la atención en tratar de saber cómo y por qué los miembros de una organización crean significados compartidos, afectaciones de los sistemas y supra sistemas y la evolución y cambios a través del tiempo, todo ello por la comunicación (Eco, 2002). En I990, las organizaciones se preocupan por orden y progreso, por un equilibrio entre la vida laboral y las familias (Eisenberg y Goodall, 2003).

A partir del año 2000, la comunicación organizacional posibilita la contribución de mantener activas a las personas que integran las organizaciones, se da la apertura para el diálogo ante las áreas de oportunidad o problemas, tratando de dar una solución consensuada que beneficie la producción (Rebeil y Ruizsandoval, 2000). Otro aporte que enriquece esta época es la propuesta de Villafañe (2013), en la que la comunicación para las organizaciones se puede percibir desde el lenguaje, ya que los signos de cada organización representan la identidad corporativa y la cultura organizacional. La identidad corporativa entendida como los aspectos que a través de la historia de la organización le han dado personalidad y carácter (Mayol, 20IO). En otra postura, Guillen y Espinosa (20I4) refieren que es una manera de producir y hacer intercambio de significados que posibilitan el flujo de mensajes que dan sentido y orden de manera interna y externa en las organizaciones, esto fortalecerála cultura organizacional y el logro de los objetivos.

Actualmente, ante los diferentes enfoques y percepciones de los investigadores y especialistas, es importante destacar que esta disciplina también se la ha denominado comunicación institucional, comunicación corporativa, comunicación interna, relaciones públicas, comunicación integral para las organizaciones, entre otros (Rebeil y Moreno, 20I4). La comunicación organizacional, menciona Rebeil (2006), tiene tres dimensiones: comunicación institucional (imagen), comunicación interna (gestión) y comunicación mercadológica (ventas). Estas dimensiones posibilitan que los colaboradores, las estrategias y líderes actúen en congruencia con la visión, los valores y la cultura como parte de la comunicación corporativa. Por otra parte, la comunicación organizacional actualmente no se puede concebir sin el uso de la tecnología, como una fortaleza que pone a las organizaciones a la vanguardia frente a otros sistemas que viven cambios de tipo social, económico y/o político. En este contexto, el especialista está obligado a saber percibir las áreas de oportunidades, comprender los cambios, prospectar los problemas, decodificar mensajes e inducir la motivación y el silencio, según sea necesario (Rebeil, 2006). 


\section{EL USO DE LA METODOLOGía DE LA INVESTIGACIÓN PARA \\ LOS ESPECIALISTAS DE LA COMUNICACIÓN ORGANIZACIONAL}

Los métodos de investigación hacen referencia a números y cualidades, sin embargo, cuentan con elementos que los complementan y van mucho más allá de esa expresión simplista, los elementos permiten descubrir el por qué y la razón de ser de los procesos en estudio. La metodología de la investigación es una herramienta que en algún momento de la vida académica del especialista de la comunicación organizacional tendrá que usar porque para la aplicación previa de herramientas de comunicación se necesita un estudio anterior con rigor científico que ofrezca resultados válidos.

También a la metodología de la investigación se le puede definir como el área del conocimiento que coordina las técnicas y pasos a seguir durante el desarrollo de un proceso de investigación que busca un objetivo y genera conocimiento. Asimismo, esta disciplina contribuye en focalizar lo que se quiere investigar y la forma en que se recolectan, analizan y clasifican los datos, que tendrán validez y pertinencia en la investigación que se realiza (Lafuente, Carmen y Egoscozábal, Marín, 2008).

No obstante, la investigación científica determina el método a utilizar, dependiendo del fenómeno a estudiar, de los objetivos a alcanzar y el tipo de análisis a realizar. Hay dos métodos más generales, el método cuantitativo y el método cualitativo; el primero busca dar una respuesta numérica a través del uso de encuestas, estudios demográficos, inventarios, análisis estadístico. El segundo método que da resultados desde diferentes perspectivas ofrece respuestas que son resultado de análisis, entrevistas, trabajo de grupos, observación, descripción de hechos (Nateras, 2005).

La metodología para el desarrollo de proyectos de intervención en la línea terminal de comunicación organizacional de la UPAEP que utilizan los estudiantes puede ser cualitativa, cuantitativa o mixta, se induce a los universitarios a realizar un estudio de los procesos sociales que viven las organizaciones públicas o privadas con la intención de que a través de la investigación comprendan de mejor manera a las personas y a sus contextos. Además de aprender a hacer uso de todos los instrumentos y técnicas científicas que ocuparán en las organizaciones con la que podrán alcanzar los objetivos y conocer sus necesidades, áreas de oportunidad, la manera en que se deberá diseñar la comunicación que va dirigida a los colaboradores y a los sistemas externos, los efectos que tiene en ellos, así como la forma en que sus integrantes adoptan y viven la filosofía organizacional. 


\section{PROYECTOS DE INTERVENCIÓN EN LA LÍNEA TERMINAL} DE COMUNICACIÓN ORGANIZACIONAL DE LA UPAEP

La Universidad Popular Autónoma del Estado de Puebla (UPAEP) se fundó en I973, el programa en Ciencias de la Comunicación se impulsó en I983 ante la demanda de las necesidades sociales del estado de Puebla. Hasta la fecha seis han sido las actualizaciones que ha tenido el plan de estudios, la más actual es la de 2013, plan 02. En 2008 se modificó el nombre a licenciatura en Comunicación constituyéndose el plan or. Ante los constantes cambios sociales y las transformaciones tecnologías que día a día se experimentan en el mundo indujo a repensar el enfoque del programa de estudios sobre Comunicación en la UPAEP, por lo que en el año de 1995 se buscó ampliar la formación de los especialistas en comunicación mediante cursos complementarios sobre comunicación organizacional, comunicación educativa, mercadotecnia, entre otras. En el plan o (2008) se incorporan líneas terminales en la currícula: Comunicación Política, Comunicación Organizacional y Comunicación para el Desarrollo, con la intención de incidir en otros campos laborales. En el plan o2 se continúan las líneas terminales. Actualmente existe un programa con énfasis en el manejo del software y las plataformas digitales, para la realización de productos multimedia digitales, esto no exime que los estudiantes cuenten con una formación en teorías de la comunicación, conocimiento del contexto social, carácter reflexivo y análisis crítico, a fin de generar contenidos de calidad.

El interés de la UPAEP porque sus estudiantes conocieran más acerca de la disciplina data del año 2000, donde la Dra. Margarita Argüelles impulsa a manera de formación extracurricular un Diplomado en Comunicación Organizacional, asesorado y guiado por académicos de la Universidad Anáhuac Norte: Mtro. José de Jesús González Almaguer y el Mtro. Juan Andrés del Rincón (actualmente ya no laboran en esa casa de estudios), quienes eran parte de la planta académica del Diplomado, era la UPAEP, a través de su programa en Ciencias de la Comunicación la primera institución en la ciudad de Puebla en promover este tipo de conocimiento.

Los proyectos de intervención en Comunicación Organizacional que realizan los estudiantes de la UPAEP realmente inician en el año 2004 con la asignatura de Taller de Comunicación Organizacional, el implementar proyectos en organizaciones públicas y privadas se da como una iniciativa académica de Eva María Pérez Castrejón, titular de la asignatura y directora del programa académico en esa fecha, ante la latente necesidad de que los universitarios combinaran los conocimientos teóricos con los prácticos. En 2008 con el rediseño curricular y el cambio de nombre del programa a Comunicación, se formaliza el desarrollo de proyectos de intervención en la línea, aplicados con más profesionalismo y precisión teórico-práctica en organizaciones públicas y privadas por los estudiantes. 
Para el 2012 se tuvo la primera generación de la línea terminal de Comunicación Organizacional, Karina Torrescano se integra como docente de tiempo completo del programa en Comunicación, cuyas funciones eran impartir asignaturas de la línea y coadyuvar a los esfuerzos realizados previamente.

Actualmente el desarrollo de proyectos se continúa realizando a pesar de no contar con líneas terminales en el programa.

FORMALIZACIÓN DE LA LÍNEA TERMINAL

DE COMUNICACIÓN ORGANIZACIONAL EN LA UPAEP

Para Enrique B. Franklin los manuales administrativos son "documentos que sirven como medios de comunicación y coordinación que permiten registrar y transmitir en forma ordenada y sistemática, información de una organización (antecedentes, legislación, estructura, objetivos, políticas, sistemas, procedimientos, etc.), así como las instrucciones y lineamientos que se consideren necesarios para el mejor desempeño de sus tareas". Para el 2014 se generó un manual propio de la línea que incluía: objetivos, perfil de egreso, conceptos básicos, asignaturas impartidas, proceso para la selección de empresa, información de las asignaturas de diseño, evaluación de proyectos y seminario, opciones de titulación y anexos útiles como cartas de aceptación para realización de proyectos de intervención por parte de las empresas, carta de inicio de prácticas profesionales y carta de término/liberación de prácticas de los estudiantes inscritos en la línea. Dicho manual buscó formalizar y homologar información relacionada con la línea, necesaria para los alumnos que debían realizar un proyecto de intervención. De esta manera, el contenido del manual daba a conocer a los alumnos los requisitos necesarios e indispensables para el desarrollo de proyectos de intervención. Además, dicha información se anexaba a la guía de aprendizaje y se les hacía saber desde la primera clase y reunión con los alumnos de la línea. Al finalizar cada uno de los semestres de las asignaturas de diseño, estrategia y evaluación de proyectos, los alumnos debían presentar sus avances según el cronograma establecido ante los representantes de las organizaciones donde realizan sus proyectos.

\section{METODOLOGÍA DE PROYECTOS DE INTERVENCIÓN EN COMUNICACIÓN}

A partir del último cuarto de siglo, quedó claro que el estudio del fenómeno comunicativo tiene un origen multidisciplinario, aportaciones teóricas y metodológicas de las diferentes 
disciplinas permitieron en un primer momento comprender y analizar aquellas prácticas que estaban relacionadas con la comunicación. El objetivo del análisis comunicacional implica, por tanto, la adquisición de conocimientos y la elección de métodos adecuados que permitan comprender la realidad; son esos métodos, para la subacademia de intervención con énfasis en la línea organizacional en casos de Comunicación, de suma importancia y el eje del presente informe.

Por lo que el uso de métodos cualitativos y cuantitativos ha generado profundos debates sobre “¿cómo?” “ “ipor qué?” debe usarse uno u otro; sociólogos, antropólogos, psicólogos, estadistas, entre otros, analizan qué paradigma sería el adecuado para las ciencias sociales. Y es que, desde un inicio, los avances del pragmatismo en las ciencias naturales motivaban el deseo epistémico de aplicar el método a las nacientes ciencias sociales. Autores como Spencer, Comte, Saint-Simon y John Stuart Mill, planteaban la necesidad de seguir el camino naturalista, a riesgo de generar investigaciones carentes de conocimiento, que no aportarían comprensión o explicación del mundo. La ciencia social se interesó, en un primer momento, por lo general y lo universal, el objetivo era estar fundamentado desde la objetividad científica, buscando siempre comprender la realidad desde la perspectiva del observador externo.

Así, la metodología basada en lo cuantitativo tiende a agrupar a los individuos a partir de características que permiten homogeneizar el todo en variables para cuantificar a través de inducción probabilística, medición controlada, elementos puramente objetivos, análisis de datos duros y repetibles, así como la generalización de resultados. Los académicos e investigadores que estudian el fenómeno comunicativo no quedaron fuera de dicha confrontación y durante las décadas de los años cuarenta, cincuenta y sesenta la metodología de investigación se enfocó en el uso de los métodos cuantitativos. Un ejemplo claro fueron los estudios realizados por la Mass Comunication Reserch, fuertemente criticados por sus posturas epistemológicas, teóricas e ideológicas, que generaron una corriente de pensamiento caracterizada por una visión positivista, la cual buscaba medir el impacto de los procesos comunicacionales a través de los efectos producidos por los medios masivos de información.

Algunos de los aportes teóricos y metodológicos durante esta etapa se basaron en esquemas funcionalistas, por ejemplo, lo planteado desde la psicología experimental norteamericana, que dirigía sus esfuerzos para comprender a las audiencias, en específico, el impacto de los mensajes difundidos por los medios masivos de información, medido a partir de los efectos que supuestamente producían a los individuos que se sometían a ellos. Estudios cuantitativos que medían la opinión pública se popularizaron, por la necesidad de las empresas que buscaban conocer la cantidad de individuos que se sometían a ciertos mensajes, bajo el supuesto de que así lograrían incrementar las ventas de sus productos. Se generó la idea de que todo individuo, sin importar su contexto, es parte de un público mundial, y por 
ende es parte de la masa, que lo dota de símbolos referenciales comunes y que a partir de dichos símbolos comunes, lograrían participar de manera efectiva en la actividad pública de cualquier parte del globo.

Sin embargo, al inicio de la década de los ochenta se notó la limitación del modelo cuantitativo (funcionalista) antes mencionado, si bien se buscaron nuevas formas de comprender la realidad social, no se omitieron los métodos cuantitativos. Las críticas planteadas al positivismo, su metodología y su concepción del mundo no son nuevas, ya Max Weber (1984) planteó la necesidad de hacer una distinción metodológica entre las ciencias sociales y las ciencias naturales. Una de las principales diferencias planteadas por Weber habla de la necesidad de utilizar la "interpretación" para captar la acción social y, a partir del recurso metodológico de la "empatía", generar un análisis de la realidad más completo y complejo, explicando de manera causalmente la acción social, en nuestro caso el fenómeno comunicativo.

Aun cuando, en momentos más recientes, se pasó del funcionalismo norteamericano a la teoría crítica planteada por la Escuela de Frankfurt que calificaba como mercantilista a los medios de información, dicho enfoque criticaba el servicio de los medios para la imposición de los valores de las clases dominantes. La Escuela de Frankfurt y la Escuela Francesa, además de modificar los preceptos teóricos, también motivaron el cambio de paradigma metodológico, dando más importancia a las técnicas cualitativas. Dicha discusión replanteó la necesidad de un proceso en el cual la investigación cualitativa necesariamente desarrolle nuevas técnicas de recopilación y análisis del material empírico, así como la fundamentación teórica de esta nueva perspectiva metodológica, además de la producción de reflexiones que permitan la comprensión del fenómeno comunicativo de manera más profunda a partir de la construcción de conocimiento.

Por lo que, esta nueva estrategia metodológica basada en un enfoque crítico-hermenéutico buscaba la comprensión de la realidad de los sujetos haciendo énfasis en la comprensión de su contexto, tomando como referencia el análisis del discurso, sus percepciones, las vivencias y experiencias de los individuos. Ahora se producen datos descriptivos, que incluyen las palabras de las personas así como la conducta observable. Quedó claro entonces que sería difícil comprender a este nuevo mundo lleno de características tan diferentes, tanto en aspectos económicos, políticos, sociales y culturales a partir de la objetividad positivista.

Así, creemos que la propuesta cualitativa de investigación está reformulando el paradigma metodológico en el quehacer del comunicólogo, convirtiéndose en una tendencia dominante dentro de los estudios del fenómeno comunicativo. Cada vez está más claro que la sociedad es múltiple, que se construye y redimensiona a partir de la interacción entre los individuos y el entorno, por lo tanto las propiedades emergentes de la misma se modifican y modifican los sentidos de lo "ya establecido", así incluso la investigación cualitativa nece- 
sitará replantearse epistemológica y metodológicamente para poder explicar las tendencias comunicacionales del futuro.

Esta formación emergente construida por la subacademia de Intervención de Proyectos de Comunicación partiría de modelo de la Comunicometodología, es decir, una metodología para el diseño de proyectos de intervención desde la planeación estratégica, la cual parte de una proyección programática de una serie de acciones que deberán ser gestionadas a partir de sus objetivos, para, posteriormente ser evaluada, reconstruida e implementada. Así, al hablar de intervención de un Proyecto de comunicación, en una realidad comunicativa específica, estaremos hablando de la capacidad del profesional para diagnosticar problemas o identificar áreas de oportunidad, diseñar una estrategia, gestionar o desarrollar los productos necesarios de dicha estrategia, para después evaluarla y así generar experiencia; dicha experiencia se traducirá en conocimiento que le permita continuar interviniendo en la sociedad y generar cambios en la misma.

\section{PROPUESTA DEL MODELO DE PROYECTOS DE INTERVENCIÓN}

\section{Ruta crítica (I). Metodología para proyectos de intervención}

Basado en la propuesta del Reglamento de Titulación de la Licenciatura en Comunicación UPAEP (2OIO)

\section{Ideas base:}

- La intervención, desde la planeación estratégica, propone que un proyecto es una definición programática.

- Toca de una acción que deberá ser gestionada a partir de sus objetivos, evaluada, reconstruida y por último aplicada.

- La intervención de un proyecto se realiza para diagnosticar problemas e identificar áreas de oportunidad (Comunicación Personal. Asesoría. Junio 2012. Universidad Popular Autónoma del Estado de Puebla).

- Martín Serrano establece ejes, los de comunicología interacción, difusión, expresión y estructuración, que permiten investigar y diagnosticar sistemas que permiten identificar prioridades en un determinado entorno (Macías y Cardona 2007). 


\section{ESTRUCTURA METODOLÓGICA}

\section{La detección}

Generar ideas potenciales para investigar, plantear el problema de investigación. Asignatura: Diseño de proyectos de Comunicación.

\section{Contenido:}

- Registro del fenómeno

- Antecedentes de la situación problemática

- Delimitación del sistema

- Construcción conceptual

- Determinación de macros y micros

- Análisis ambiental

- Antecedentes de información

- Elaboración de hipótesis

\section{Investigación}

Identificar problemas a ser resueltos o áreas de oportunidad para ser abordados. Cuali-cuanti-enfoque mixto.

- Metodología de investigación

- Técnicas de investigación

- Resultados de investigación

\section{El diagnóstico}

Asignatura: Estrategia de Proyectos de Comunicación.

\section{Contenido:}

- La enunciación

- Cuadros de clasificación del diagnóstico

- Comprensión de los sujetos de interacción 


\section{El plan estratégico de comunicación}

Elaborar escenarios. Qué se quiere o qué no se quiere lograr.

- Establecer objetivos

- Estrategias y tácticas (primera fase)

- Mensaje

- Público

- Vocero

- Medios

- Cronograma de acciones comunicativas

- Tiempos y estrategias de evaluación

\section{I. Gestión}

Asignatura: Evaluación de proyectos.

Contenido:

Objetivo:

- Práctica del proyecto a partir de los objetivos

- Producción de medios

\section{Plan estratégico para la intervención}

Metodologías de evaluación.

Diseño de evaluación

- Costo efectividad

- Funcionalidad

- Cumplimiento de metas

- Construcción de escenarios

Posteriormente, con la incorporación de Eva María Pérez Castrejón al Doctorado en Investigación y Comunicación Aplicada, se tomó en cuenta la subdisciplina de la Comunicación, 
denominada como la Comunicación Aplicada, entendida esta como el estudio teórico y metodológico de los procesos de comunicación a la luz de fundamentos conceptuales y metodológicos científicos, con el fin de buscar la verdad y además proponer vías de solución aplicables a los problemas de individuos, grupos, organizaciones, comunidades y sociedades específicas, sean éstos de la comunicación interpersonal o de la colectiva.

Estudios sobre epistemología de la comunicación aplicada en el ámbito laboral, así como sobre comunicación estratégica o integral aplicada para las micro, pequeñas y medianas empresas, así como para organizaciones públicas y de la sociedad civil (Fuentes, 20II; Rebeil, 20II).

Asimismo, el impulso a la investigación, a partir de demostrar cómo sus resultados son aplicables a la solución de problemas de la sociedad y de las organizaciones y los grupos, significa un paso ineludible que pueden y deben dar más investigadores de la comunicación. Las aportaciones no solamente están en la ampliación de la comprensión de los fenómenos y en la producción de datos, estadísticas o explicación de los fenómenos, sino también en proveer soluciones a la medida. Así es como la Comunicación Aplicada revela información sustantiva y significativa acerca del problema social que está analizando (Arévalo, Moreno y Rebeil, 2012).

La base de la Comunicación Aplicada son los planteamientos teóricos de fondo, no obstante, su propósito no queda en la producción de bases conceptuales y teóricas que permiten la descripción y la explicación de los fenómenos sociales. Los trabajos de investigación de la Comunicación Aplicada inician con la investigación básica pero su fin anterior es generar conocimiento que permita abordar los problemas del mundo real. Los investigadores de la Comunicación Aplicada reconocen que "la investigación pura es una fase preliminar de la investigación aplicada” (Ander-Egg, 2003).

Las aportaciones de Macías y Cardona del modelo de comunicometodología y las de Arévalo y Rebeil de comunicación aplicada, junto con estrategias de formalización para el desarrollo de proyectos de intervención, establecieron el modelo aplicado en los diferentes proyectos de intervención que realizan los estudiantes de esta casa de estudios en organizaciones públicas y privadas, con el fin de vincular su formación en la línea con sus prácticas profesionales y experiencia laboral; además de reforzar el desarrollo teórico en congruencia con la práctica universitaria.

Cabe mencionar que la vinculación con los organismos referentes y representativos de la comunicación organizacional como la Asociación Mexicana de Comunicadores (AMCO) y la Asociación Mexicana de Investigadores y Profesionales de la Comunicación (AMIPCO), ha sido necesaria para la conformación y solidificación del modelo, ya que con la primera mencionada siendo miembros de la AMCO nos brindan servicio con el fin de impulsar a 
los comunicadores a ubicarse dentro de sus organizaciones como aliados estratégicos en el logro de los objetivos del negocio, así como a potenciar la función incorporando rutinas de evaluación y medición en un proceso de mejora continua. Esto nos ha permitido detectar las necesidades reales y emergentes de las distintas organizaciones, además de sumar a los alumnos a viajes de estudio cuyo fin único es conocer y participar con las áreas de comunicación de las distintas organizaciones. La capacitación y formación extracurricular con esta asociación cada vez es más fuerte, ya que al igual que la AMIPCO, los presidentes de ambos organismos son miembros del actual consejo de vinculación del programa de comunicación y medios digitales de la UPAEP.

\section{METODOLOGÍA O DATOS PRINCIPALES DEL CASO}

La metodología de investigación que se utiliza es cuantitativa, ya que se emplearon técnicas como: análisis y reportes de bases de datos. Este tipo de metodología es resultado de un estudio cuantitativo ya que muestra el control sobre el fenómeno a investigar y posibilita un conteo de los resultados. En esta investigación se diseñó un instrumento para la recolección de datos que consistió en documentar información de los egresados y de las dos últimas generaciones de alumnos. El marco muestral son los egresados de la licenciatura en Comunicación de la línea terminal en comunicación organizacional de la UPAEP, que del 2012 al 2017 suman 55 alumnos, así como los nueve alumnos que cursaron la asignatura de diseño de proyectos en los últimos dos años con proyectos organizacionales.

Se realizó un análisis de las bases de datos de los repositorios de la misma casa de estudios y se tomó en cuenta el testimonio de las dos autoras de este informe. La investigación cuantitativa obtiene resultados lógicos, numéricos y estadísticos, como procedimiento que establece las magnitudes numéricas. Se apoya del canon que menciona: las partes representan el todo; estudiando una muestra nos da la idea de cómo está la población en su conjunto (Hueso y Cascant, 20I2).

En la Gráfica i se muestra el total de alumnos de las seis generaciones del plan or y o2 del programa de comunicación con opción de líneas terminales, que nos permite visualizar el porcentaje global con $48 \%$ de alumnos egresados de la línea terminal de comunicación organizacional, $34 \%$ de comunicación para el desarrollo y $21 \%$ de comunicación política. 


\section{GRÁFICA 1. TOTAL DE ALUMNOS DE LAS SEIS GENERACIONES}

Programa académico: Comunicación

Plan de estudios: or y 02

Líneas terminales

Generación: Otoño 2012-Otoño 2017

\begin{tabular}{|c|c|c|c|}
\hline GENERACIÓN & $\begin{array}{c}\text { COMUNICACIÓN } \\
\text { PARA EL } \\
\text { DESARROLLO }\end{array}$ & $\begin{array}{l}\text { COMUNICACIÓN } \\
\text { ORGANIZACIONAL }\end{array}$ & $\begin{array}{l}\text { COMUNICACIÓN } \\
\text { POLÍTICA }\end{array}$ \\
\hline Generación 2008-2012 & 10 & 19 & 4 \\
\hline Generación 2009-2013 & 11 & 7 & 6 \\
\hline Generación 2010-2014 & 6 & 9 & 3 \\
\hline Generación 2011-2015 & 10 & 7 & 2 \\
\hline Generación 2012-2016 & 2 & 7 & 1 \\
\hline Generación 2013-2017 & 0 & 6 & 5 \\
\hline Total de alumnos & 39 & 55 & 21 \\
\hline \multicolumn{2}{|c|}{$\begin{array}{c}\text { Comunicación } \\
\text { políitica } \\
18 \%\end{array}$} & \multicolumn{2}{|c|}{$\begin{array}{c}\text { Comunicación } \\
\text { para el desarrollo } \\
34 \%\end{array}$} \\
\hline
\end{tabular}

FUENTE: ELABORACIÓN DEL AUTOR CON DATOS DE LA DIRECCIÓN DE EFECTIVIDAD INSTITUCIONAL DE LA UPAEP.

En la Gráfica 2 se muestra el total de alumnos de las dos últimas generaciones del plan 03 del programa de comunicación y medios digitales sin opción de líneas terminales, que nos permite visualizar el porcentaje con $47 \%$ de alumnos que realizan proyectos con temáticas organizacionales mientras que $53 \%$ con temáticas de incidencia social. 


\section{GRÁFICA 2. TOTAL DE ALUMNOS DE LAS DOS ÚLTIMAS GENERACIONES DE ALUMNOS} QUE CURSARON LA ASIGNATURA DE DISEÑO DE PROYECTOS

Programa académico: Comunicación y medios digitales

Plan de estudios: 03
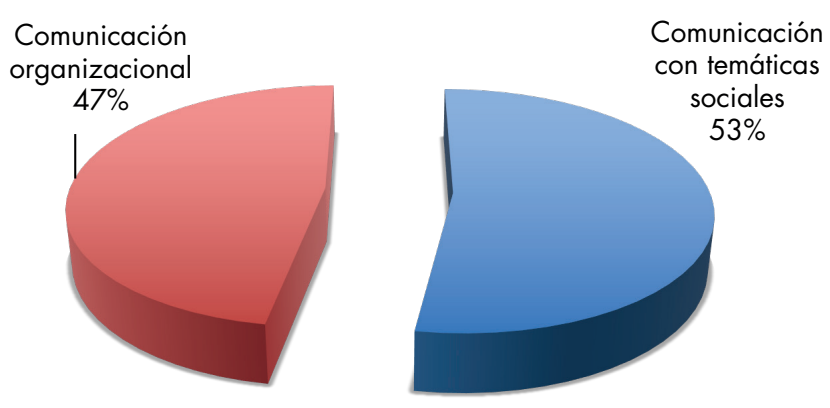

\begin{tabular}{|l|c|c|}
\hline \multicolumn{1}{|c|}{ GENERACIÓN } & $\begin{array}{c}\text { COMUNICACIÓN CON } \\
\text { TEMÁTICAS SOCIALES }\end{array}$ & $\begin{array}{c}\text { COMUNICACIÓN } \\
\text { ORGANIZACIONAL }\end{array}$ \\
\hline Generación 2014-2018 & 5 & 5 \\
\hline Generación 2015-2019 & 5 & 4 \\
\hline Total de alumnos & 10 & 9 \\
\hline
\end{tabular}

FUENTE: ELABORACIÓN DEL AUTOR CON DATOS DE LA DIRECCIÓN DE EFECTIVIDAD INSTITUCIONAL DE LA UPAEP.

En la Gráfica 3 se muestra la eficiencia de titulación (Titulación por proyectos de intervención y su aporte con la investigación). 
GRÁFICA 3 . ESTADÍSTICAS DE LAS ÚLTIMAS CINCO GENERACIONES

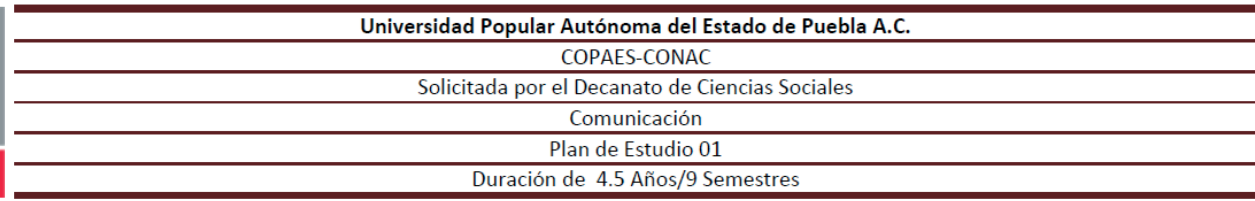

Estadísticas últimas 5 Generaciones

\begin{tabular}{|r|c|c|c|c|c|c|c|}
\hline & $2008-2012$ & $2009-2013$ & $2010-2014$ & $2011-2015$ & $2012-2016$ & TOTAL & $\%$ \\
\hline No. Ingresos & 53 & 46 & 38 & 39 & 35 & $\mathbf{2 1 1}$ & \\
\hline Mujeres & 34 & 34 & 25 & 28 & 17 & 138 & $65 \%$ \\
\hline Hombres & 19 & 12 & 13 & 11 & 18 & 73 & $35 \%$ \\
\hline $\begin{array}{r}\text { Egresados Sin Titular } \\
\text { Egresados Titulados }\end{array}$ & 2 & 0 & 3 & 7 & 5 & 17 & $16 \%$ \\
\hline Total Egresados & 33 & 24 & 15 & 12 & 5 & 87 & $84 \%$ \\
\hline
\end{tabular}

16\% Egresados Sin Titular

Egresados Titulados

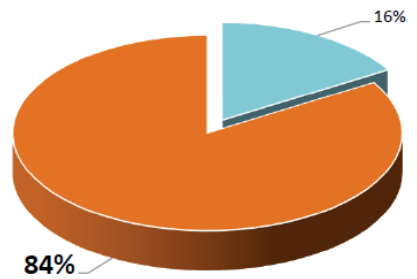

Análisis Longitudinal (Análisis de la información a través del tiempo en forma acumulada)

\begin{tabular}{|c|c|c|c|c|c|c|c|c|}
\hline $\begin{array}{c}\text { Año de Ingreso } \\
* * \text { (Generación) }\end{array}$ & $\begin{array}{c}\text { Egresados en } \\
\text { tiempo }\end{array}$ & $\begin{array}{c}\text { Egresados } \\
\text { 1er Año }\end{array}$ & $\begin{array}{c}\text { Egresados } \\
\text { 2do Año }\end{array}$ & $\begin{array}{c}\text { Egresados } \\
\text { 3er Año }\end{array}$ & $\begin{array}{c}\text { Titulados } \\
\text { 1er Año }\end{array}$ & $\begin{array}{c}\text { Titulados } \\
\text { 2do Año }\end{array}$ & $\begin{array}{c}\text { Titulados 3er } \\
\text { Año }\end{array}$ & $\begin{array}{c}\text { Titulados 4to } \\
\text { Año }\end{array}$ \\
\hline $2008-2012$ & 12 & 28 & 32 & 33 & 17 & 22 & 29 & 31 \\
\hline $2009-2013$ & 12 & 20 & 22 & 24 & 12 & 16 & 22 & 24 \\
\hline $2010-2014$ & 7 & 16 & 18 & 18 & 9 & 15 & 15 & NA \\
\hline $2011-2015$ & 7 & 19 & 19 & NA & 10 & 12 & NA & NA \\
\hline $2012-2016$ & 10 & 16 & NA & NA & 5 & NA & NA & NA \\
\hline
\end{tabular}

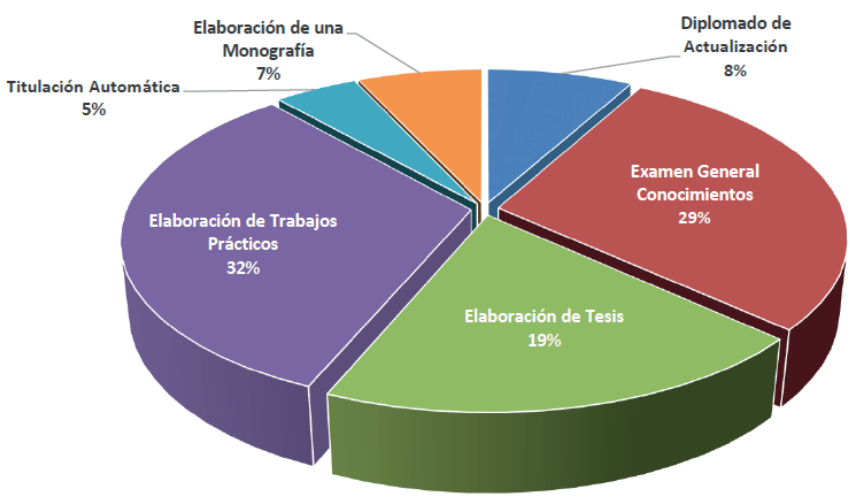

Dirección de Efectividad Institucional

Elaboró: Mtra. María Elena Lara Rodríguez

Resumen Estadístico Egresados

FUENTE: ELABORACIÓN DEL AUTOR CON DATOS DE LA DIRECCIÓN DE EFECTIVIDAD INSTITUCIONAL DE LA UPAEP. 


\section{RESULTADOS O DESARROLLO DEL CASO}

La población estuvo conformada por un total de 64 alumnos que desarrollaron o están por concluir su proyecto de intervención con énfasis en comunicación organizacional, de los cuales 55 son ya egresados del programa y nueve son alumnos que aún se encuentran estudiando o están por egresar. En un primer momento se analizaron las bases de datos generadas por la Dirección de efectividad institucional y por la Dirección académica del programa de estudios de la licenciatura en Comunicación y medios digitales. Esto permitió identificar los 55 alumnos inscritos en la línea organizacional en CO del plan or y o2 de un total de II5; posteriormente se obtuvo el número de los nueve estudiantes que han tenido interés en la disciplina de los ig alumnos que están por egresar de las dos últimas generaciones. Siendo que la totalidad de la muestra ha realizado proyectos aplicados en organizaciones públicas y privadas por año y en total de todos los periodos.

\section{DISCUSIÓN O ANÁLISIS DEL CASO}

Los resultados facilitan percibir el nivel de efectividad de la propuesta curricular de la línea terminal y el nivel de incidencia de los estudiantes en sistemas externos, lo que permite establecer gráficamente el modelo utilizado. La aplicación de proyectos de intervención posibilita que los universitarios desarrollen competencias y conocimientos profesionales a través de los cuales han incidido de manera más efectiva en el ámbito laboral.

Por lo que el especialista en comunicación organizacional de la UPAEP es un profesional que tiene conocimientos y bases teóricas que le permiten incurrir en la práctica organizacional, una vez que investigan las necesidades particulares para organización; pueden diseñar piezas de comunicación específicas a las necesidades de cada organización, posteriormente aplicarlas y finalmente evaluarlas. Lo antes mencionado es ejecutado teniendo como referente la identidad corporativa a partir de una filosofía organizacional ya establecida.

\section{CONCLUSIONES}

El presente escrito hace un breve resumen de los tipos de investigación establecidos por las Ciencias sociales y de su evolución y adecuación con la disciplina de la Comunicación. Están contemplados en la metodología para el desarrollo de proyectos de intervención en comunicación organizacional, porque facilita la claridad sobre el objetivo central del es- 
crito; el cual se orienta a provilegiar aquellos estudios que nos arrojan una visión integral de la comunicación en las organizaciones. De este modo destaca: la psicología, sociología, administración, economía, antropología social y mercadotecnia y publicidad, que sin lugar a dudas, el aporte de otros pensadores como sociólogos y psicólogos han enriquecido el campo y la subdisciplina. El resultado que se puede aducir es que al abordar organizaciones e intervenir en ellas, las metodologías para recolectar información y analizarlas se materializan en una multiplicidad de técnicas, cada una de ellas con sus especificidades. En todos los casos, lo que se intenta es tender a la rigurosidad y a mostrar límites y recursos, y evitar el espontaneísmo (Contreras, 1993).

No hay técnicas de recolección de información ni herramientas que sean mejores que otras. La elección, uso y aplicación de alguna de ellas derivan de los objetivos propuestos tanto en el proceso mismo de intervención, como en la justificación del uso de la técnica y la herramienta. En este trabajo nos concentramos en exponer la metodología adecuada a las necesidades del programa y de los estudiantes. Así como de describir los retos, áreas de oportunidad y resultados obtenidos.

El artículo no muestra medición de resultados, ya que como se menciona, es un recorrido histórico de las líneas de enfoque y del interés profesional del perfil de egreso que la UPAEP ha tenido para sus estudiantes y egresados que inciden en organizaciones públicas y privadas. Asimismo, se muestran resultados de la eficiencia terminal de la línea en comunicación organizacional, donde los estudiantes adquieren habilidades y competencias propias de la disciplina, mismas que a partir del año primer lustro del 2000 se han puesto en práctica dentro de organizaciones públicas y privadas con la intención de medir y conocer la efectividad.

La aplicación de las metodologías mencionadas en el texto posibilitó que los académicos que imparten las asignaturas asociadas a la disciplina, desarrollaran contenidos que a los estudiantes les dieran más herramientas no solo en las aulas, sino en la práctica con sistemas externos y así poder migrar de lo teórico a lo práctico. Las metodologías implementadas en el programa académico de comunicación de la línea terminal en comunicación organizacional de la UPAEP posibilitó que los estudiantes no se quedaran con saber elaborar o implementar un diagnóstico o pensar en piezas de comunicación en un soporte material, sino que obtuvieron los conocimientos necesarios para diseñar e implementar un diagnóstico, proponer y aplicar herramientas de comunicación (plan estratégico) y medir la efectividad de las organizaciones.

Por lo que las lecciones aprendidas durante este recorrido histórico académico recaen en que los estudiantes de programas en comunicación necesitan desarrollar no solo conocimientos teóricos, sino también prácticos. Las habilidades y competencias para 
los estudiantes de comunicación se consideran imprescindibles en su formación, dichas competencias aplicadas y desarrolladas en las organizaciones permitirán que quienes forman la organización y con quienes se trabaja de manera externa tengan un ambiente de entendimiento donde se puede disminuir las áreas de oportunidad o de conflicto en materia de comunicación.

Las competencias que desarrollan los estudiantes sirven como estrategia persuasiva para llegar a públicos de manera directa e indirecta, ya que posibilitan que los especialistas sean más analistas, interpreten de mejor manera el contexto, sean creativos, sepan manejar conflictos y crisis, puedan negociar y vincular, así como mediar y solucionar a partir de la filosofía organizacional y los objetivos organizacionales.

A lo largo de la revisión del modelo aplicado en los diferentes proyectos de intervención que se ha dado cuenta en este texto, existen hallazgos que se mencionan a continuación:

La comunicación y la organización son un binomio inseparable y como profesionales de la comunicación es necesario responder a las necesidades de esta, así como aprovechar la comunicación en las organizaciones como una oportunidad de crecimiento. La formalización del modelo aplicado en los proyectos de intervención de la línea de comunicación organizacional ha impulsado la investigación a partir de mostrar cómo sus resultados son aplicables a las distintas organizaciones y necesidades sociales y/o grupales.

En la actividad cotidiana de la UPAEP habremos de formar los líderes capaces de llevar adelante las verdaderas soluciones a nuestros problemas y construyan con el pueblo un México nuevo. Por lo que se considera pertinente el modelo aplicado en los proyectos de intervención en CO de la UPAEP.

Las metodologías existentes y evolucionadas enunciadas anteriormente facilitaron la adaptación y formalización del modelo con nuestro programa e institución.

La empleabilidad de los alumnos egresados de la línea se beneficia a raíz de la realización de los proyectos de intervención, como resultado de su experiencia adquirida, así como de las relaciones y competencias adquiridas con los entornos organizacionales.

La eficiencia de titulación mejoró en $82 \%$ con el egreso de alumnos que realizaron sus proyectos de intervención y el $51 \%$ se tituló por proyectos de investigación.

Los resultados del Ceneval son mucho más satisfactorios en los alumnos que egresan por haber realizado un proyecto de intervención, debido a sus conocimientos y competencias adquiridas durante las tres asignaturas que comprenden las fases de la realización del proyecto, destacando que la mayor habilidad requerida para este tipo de evaluación es la solución de problemas.

En dicho organismo Eva María Pérez Castrejón participó en el comité académico del Ceneval, lo cual refleja la iniciativa tomada para la realización de proyectos de intervención. 
Este trabajo de investigación presenta como propuesta la importancia que significa para el especialista de comunicación organizacional tener conocimientos teóricos y prácticos para favorecer su trabajo profesional. Asimismo, se evidencian aportaciones teórico-científicas de investigaciones que contribuyen en dar un marco contextual de la trascendencia e importancia de las aportaciones que la disciplina en comunicación organizacional ha hecho a través del tiempo.

Una vez que los especialistas en comunicación organizacional tienen los conocimientos necesarios, pueden desarrollar y/o potencializar sus competencias a partir de experiencias que han adquirido y que contribuirán positivamente en sus servicios profesionales. Ya que el especialista de la comunicación organizacional de la UPAEP puede ayudar con las organizaciones públicas y privadas a detectar las áreas de oportunidad en cuanto a los procesos comunicativos de clientes internos y externos no solo de manera lineal, sino de manera trasversal con todos los diferentes sistemas.

El especialista de la línea terminal en comunicación organizacional de la UPAEP se apoya de herramientas metodológicas cuantitativas, cualitativas o mixtas, ya que no es posible sugerir la implementación de piezas, herramientas o planes estratégicos de comunicación organizacional sin una investigación previa, los instrumentos de comunicación proponen posibles soluciones a las problemáticas u áreas de oportunidad de las organizaciones.

Asimismo, el profesional de la comunicación organizacional de la UPAEP tiene presente un proceso de intervención para las organizaciones que inicia con un diagnóstico, de acuerdo con los resultados obtenidos de la investigación, diseñar y aplicar las herramientas o medios de comunicación necesarios, y finalmente evaluar el resultado y efectividad del plan estratégico, de las piezas y/o herramientas de comunicación organizacional a corto, mediano y largo plazos.

Finalmente, la opción por intervenir en, desde, con, o para una organización, supera la discusión por fundamentar que las bases teóricas del artículo correspondan al ámbito de la comunicación organizacional y no de otras áreas de la comunicación, y nos posiciona en la elección por alguna perspectiva teórico-metodológica y un posicionamiento con respecto al sujeto y las maneras y formas de abordar la organización.

\section{REFERENCIAS}

Bartoli, A. (199i). Comunicación y organización: la organización comunicante y la comunicación organizada. Comunicación y Sociedad. Recuperado de http://www.comunicacionysociedad. cucsh.udg.mx/sites/default/files/a1_10.pdf 
De la Peza, M.D. (2012). Los estudios de Comunicación: disciplina o indisciplina. Diagnóstico del proceso de Comunicación Organizacional. Ingeniería Industrial en las Organizaciones. México: Plaza y Valdés.

Franklin, E. B. (20I4). Organización de empresas: Enrique Benjamín Franklin Fincowsky. 4a. ed. México: McGraw-Hill.

González, F. (s/f). El estado del arte. Elementos generales sobre escritura de artículos. Recuperado de https://dis.unal.edu.co/ fgonza/courses/2005-II/seminario/estadoArte.pdf

Karam, T. y Cañizález, A. (20Iо). Veinte formas de nombrar a los medios masivos. Introducción a enfoques, modelos y teorías de comunicación social. Colección de textos de la comunicación, Venezuela. Recuperado de http://www.saber.ula.ve/bitstream/handle/I23456789/31682/veinte_formas.

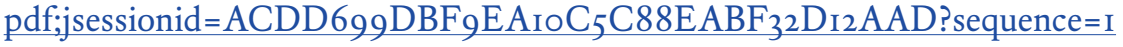

Lafuente, C. y Egoscozábal, M. (2008). Metodologías de la investigación en las ciencias sociales: Fases, fuentes y selección de técnicas. Revista Escuela de Administración de Negocios, 5-18.

Macías, N. y Cardona, D. (2007). Comunicometodología. Intervención Social Estratégica. México: UIC.

Nateras, M. E. (2005). La importancia del método en la investigación. Espacios Públicos, 277-285.

Pasquali, A. (I990). Comprender la comunicación (4a. ed.). Venezuela: Monte Ávila Latinoamericana.

Peiró, J. M. y Bresó, I. (20I2). La comunicación en las organizaciones: una aproximación desde el modelo de análisis multifacético para la gestión y la intervención organizacional (modelo Amigo). Persona, (I5), 4I-70.

Pérez, E. (8 de septiembre de 20I7). La Ética y la Responsabilidad Social Organizacional conceptos fundamentales en la formación y desarrollo profesional de los especialistas de la Comunicación Organizacional de México y España. Organicom, (ı6).

Queris, M., Almirall , A., Capote, L., y Robaina, D.A. (20I2). Diagnóstico del proceso de comunicación organizacional. Caso de estudio QUIMEFA. Ingeniería Industrial, XXXIII(2),I6I-I74. Recuperado de https://www.redalyc.org/articulo.oa?id=3604/360433580008

Rebeil, M. A. (2006). La Comunicación Organizacional en las Organizaciones. Revista Razón y Palabra.

Rebeil, M. A. y Ruizsandoval, C. (2000). El Poder de la Comunicación. México: Plaza y Valdés.

Rebeil, M.A., Arévalo, R. y Moreno, M. (2012). Comunicación aplicada: ciencia y aplicación al servicio de la sociedad. Diálogos de la comunicación, 1995-6630, I-30.

Rodríguez, R. J., Alburquerque, A. D. y Contreras, J. C. (2013). Organización e Innovación en Bibliotecas: el caso de la BDCV de El Colegio de México. Revista de Ciencias Estratégicas.

Peiró, J., y Bresó, I. (20I2). La comunicación en las organizaciones: Una aproximación desde el modelo de análisis multifacético para la gestión y la intervención organizacional (modelo Amigo).Persona, (I5), 4I-70. Recuperado de https://www.redalyc.org/articulo.oa?id=I47I/I47125259003

Universidad Popular Autónoma del Estado de Puebla (UPAEP). (20IO). Reglamento de titulación Licenciatura en Comunicación. México: UPAEP.

Weber, M. (1984). Economía y Sociedad. Conceptos de la sociología y del "significado" en la acción social. México: Fondo de Cultura Económica. 